\title{
The impact of space weather on communication
}

\author{
YURDANUR K. TULUNAY ( $\left.{ }^{1}\right)$ and PETER A. BRADLEY $\left({ }^{2}\right)$ \\ (') Faculty of Aeronautics and Astronautics, Istanbul Technical University (ITÜ), Maslak, Istanbul, Turkey \\ (2) Pandora, Scotlands Drive, Farnham Common, Slough, Berks, U.K.
}

As a preliminary to the studies conducted by the individual Working Groups, a review has been undertaken of topics which are relevant to the space-weather phenomena that can occur. The ways in general that these can influence the state of the ionosphere are discussed. The various changes in propagation parameters that arise on Earth-space and terrestrial radio paths via the ionosphere are listed. International procedures for frequency allocation and assignment are explained and the extent to which these are influenced by propagation factors is addressed. The role of mitigation techniques to improve radio-system performance is also identified.

\subsection{INTRODUCTION}

This overview was prepared (Tulunay, 2001) in response to the Action Objectives to examine briefly those present and future anticipated terrestrial and Earth-space radio systems that can be affected by space-weather phenomena. Throughout the duration of the Action emphasis has been placed on characterizing these phenomena and their associated effects. In this respect the radio systems have been taken as including all those associated with communications, navigation and surveillance. This distinction is particularly important because some phenomena, such as for example those concerned with varying signal group delays, influence the performance of navigation and surveillance systems, but are of lesser significance to systems concerned only with the transmission of intelligence. Propagation features are restricted to those arising when a radio wave travels via the Earth's ionosphere between terrestrial locations, between Earth and space, or on paths entirely within the space segment. Here the ionosphere is regarded as arising over heights from about $50 \mathrm{~km}$ upwards to several thousand kilometers and, in particular, tropospheric phenomena have been specifically excluded from consideration.

«Space weather» is a generic term involving a number of separate magnetospheric phenomena and in recent years it has become a fashionable topic for study within many branches of Solar-Terrestrial Physics (STP) (Hapgood, 2001, pers. comm.). As a result, there is a risk that the proper and necessary distinctions between space weather and STP are becoming blurred to the ultimate detriment of both topics. Space weather is concerned with understanding and where necessary mitigating the impact of timevarying phenomena in the space environment on human beings and technological systems. Many problems in ionospheric propagation clearly fall within this regime. In contrast, STP addresses the scientific understanding of the various physical regimes (e.g., ionosphere, magnetosphere and solar wind) that make up the whole space environment. It is fundamental science, much of it concerned with basic processes in plasma physics. The relationship between the two topics is perhaps best expressed by stating that STP is the body of scientific knowledge that underpins the study of space weather. Throughout 
the Action, STP has been considered only insofar as it has aided the understanding of propagation impairment effects. Likewise, optimum mitigation techniques, which are examined, can differ significantly for true two-way communication systems with the options of re-transmittal, space, frequency, time and route diversities, and other forms of error-correction procedures based on the use of intelligent modems, and for one-way navigation and surveillance radio systems, where the objective has to be to develop and deploy near-real-time propagation impairment correction algorithms.

In this section the primary types of radio systems in use in different frequency bands are surveyed within the context of international regulations (ITU, 2004). The separate propagation phenomena which can lead to system impairments, and so need to be modeled, are in turn considered. Those space-weather parameters which impact adversely and significantly on propagation conditions are identified. Mitigation techniques to overcome such problems are also addressed.

Figure 1.1 provides a schematic diagram showing the relationship between space weather and radiosystem performance (Bradley, 2003). To assess the reliability and compatibility of a radio system, i.e. whether it will achieve its intended purpose for the necessary time in the presence of the natural noise background, and whether it is compatible with co-channel and adjacent channel transmissions, appropriate propagation models are needed. These in turn depend on the relevant ionospheric models, which may be formulated in terms of either long-term predictions for system planning, or near-real-time forecasts for operational assessments. These near-real-time forecasts call for a space weather real-time database.

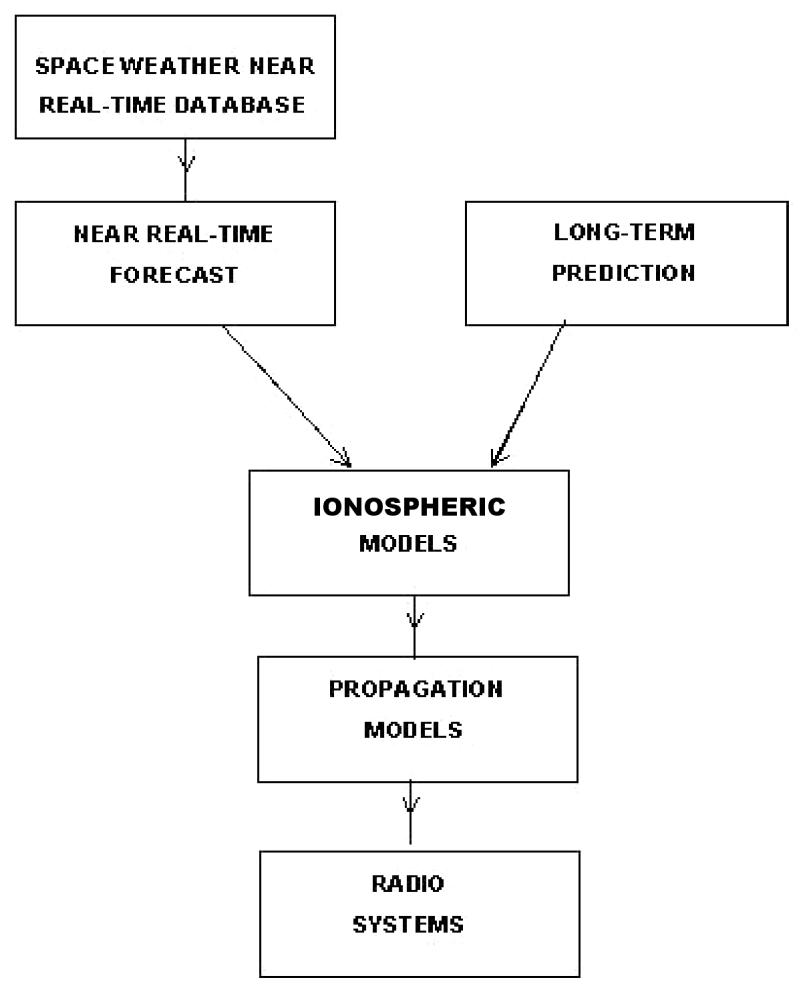

Fig. 1.1. Chart showing the chain of dependence of radio-system performance on space weather (Bradley, 2003). 
The separate sections of this Final Report confirm the progress that has been made within the Action in these respective areas, and the extent to which this information is valuable to radio users, technical commentators and decision makers.

\subsection{Present and future anticipated terrestrial and Earth-SPaCe RADIO SYSTEMS}

\subsubsection{Present usage}

International use of the radio spectrum is regulated by the Geneva-based International Telecommunication Union (ITU), a specialised agency of the United Nations in which all countries that have signed the International Telecommunications Convention of that body may participate. In so doing they undertake to introduce national legislation governing the licensing agreements of radio operating organisations within their territories which are in accord with the ITU Radio Regulations (ITU, 2004). In particular, the Radio Regulations, updated from time to time to reflect changes in system usage and requirements, lay down the conditions under which radio systems may operate. They define a number of types of radio service for varying applications, and allocate different blocks of frequencies to these individual services on the basis of operational need and propagation characteristics. Best available propagation models therefore form a key component of this process, and an important objective of COST 271 has been concerned with the refinement and enhancement of such existing models.

Article 1 of the Radio Regulations defines the separate types of radio service and Article 8 splits the world into geographical regions and zones over which separate service and frequency allocations apply. Article 8 also presents tables showing the breakdown of the spectrum into a series of frequency allocation bands for the different services, within which individual circuits having approved technical characteristics may be assigned. At the present time frequency allocation bands are specified for the overall frequency range $9 \mathrm{kHz}-275 \mathrm{GHz}$. Signals at frequencies up to 20-30 MHz, depending on conditions, rely on ionospheric reflection with potential for near-worldwide coverage after successive reflections. At VHF on frequencies that can extend to more than $100 \mathrm{MHz}$ on occasion, ionospheric support of signals radiated from ground-based transmitters is sometimes possible via scattering from ionospheric irregularities such as those responsible for the phenomena of sporadic $E$ and spread- $F$. Such signals only rarely can be used to provide a reliable service because of their intermittent incidence and the distortions that they are subjected to, but they can be an important source of interference to other co-channel services. These interference aspects have also been addressed within COST 271. Higher-frequency signals of particular interest here are mainly those involved for Earth-space paths.

The principal types of radio service defined in Article 1 which are allocated the majority of the available radio spectrum are as follows:

- The Fixed Service: a service of radiocommunication between specified fixed points.

- The Aeronautical Mobile Service: a service of radiocommunication between aeronautical stations (on the ground) and aircraft stations or between aircraft stations.

- The Maritime Mobile Service: a service of radiocommunication between coast (land) stations and ship stations or between ship stations;

- The Land Mobile Service: a radiocommunication service between base stations and land mobile stations, or between land mobile stations.

- The Broadcasting Service: a radiocommunication service intended for direct reception by the general public and which embraces both sound broadcasting and television transmissions.

- The Radiodetermination and Radionavigation Services: services using the propagation properties of radio waves to determine position, or for the purposes of navigation, etc. 
Additionally there are a number of services with lesser spectral allocations serving the needs of specialist user communities: Amateur; Meteorological Aids; Radio Astronomy; Radio Beacons; Space Research; Standard Frequency and Time Signals. The above principal services are sub-divided into component services for which different frequency allocation and technical performance specifications apply. For example, there are separate maritime and aeronautical radio-navigation services. Many of the higher frequencies are allocated to the various satellite services with specific separate bands for aeronautical mobile satellites, broadcasting satellites, earth-exploration satellites, maritime mobile satellites, meteorological satellites, radionavigation satellites and space operations. Distinctions are made between services involving fixed (geostationary) and mobile (orbiting) satellites, and between space-to-earth and earth-to-space services. There are also bands available for the amateur satellite service and for inter-satellite communication and radio-navigation services.

The Article 8 geographical regions over which different regulations and frequency allocations can apply are as shown in fig. 1.2: Region 1 covers Europe and Africa (including the former USSR), Region 2 embraces North and South America and Region 3 represents the Asian mainland together with the Australian area. In addition there is a separate Tropical Zone between latitudes of around $\pm 30^{\circ} \mathrm{N}$ and $\mathrm{S}$ of the equator where additional frequency bands between $2300-2495 \mathrm{kHz}$, as well as in three other special sub-bands in the lower part of the HF-band, are allocated to permit sound broadcasting by short-distance sky-wave signals in the presence of the large amounts of ionospheric absorption and the high thunderstorm atmospheric noise intensities encountered at such latitudes.

An example of a frequency allocation table for the frequency range $14990-18030 \mathrm{kHz}$ is given in fig. 1.3. For this frequency range, because of the worldwide nature of propagation conditions, the same allocations are adopted for all regions. Primary services are indicated with capital letters and secondary services (not accorded the same interference protection) are shown in lower case text. The

ITU RADIO REGULATIONS

Chart of Regions as Defined in Table of Frequency Allocations (See No:383 to 399 )

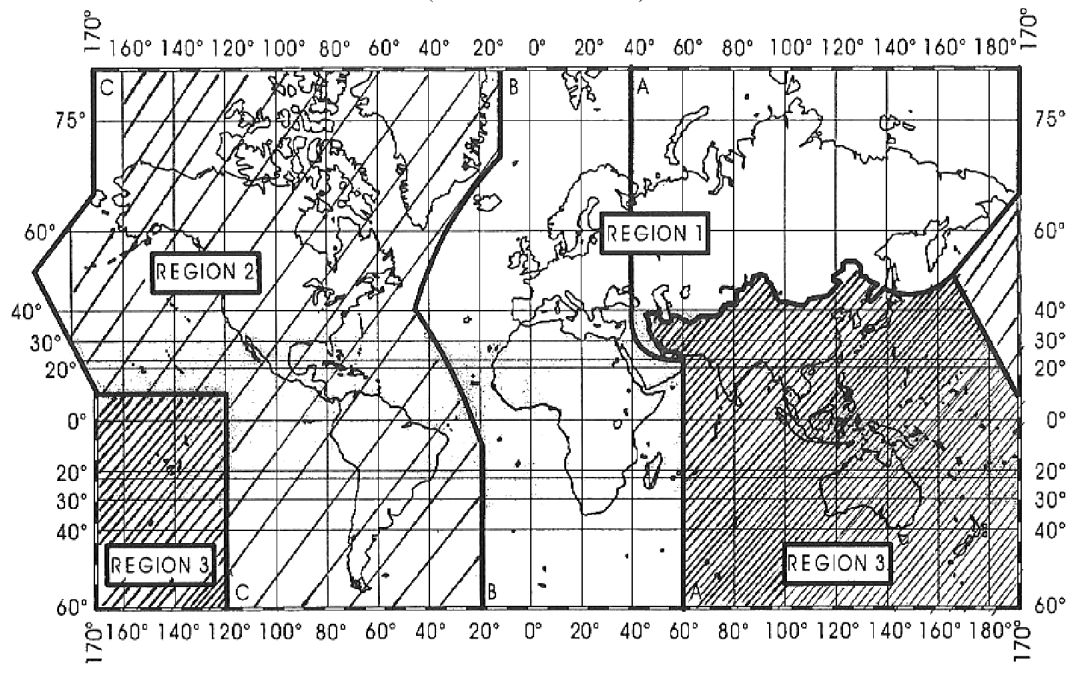

The shaded part represents the Tropical Zone as defined in No:486 to 410 and 411.

Fig. 1.2. The separate ITU geographical regions (hatched) and the Tropical Zone (dark shading). 

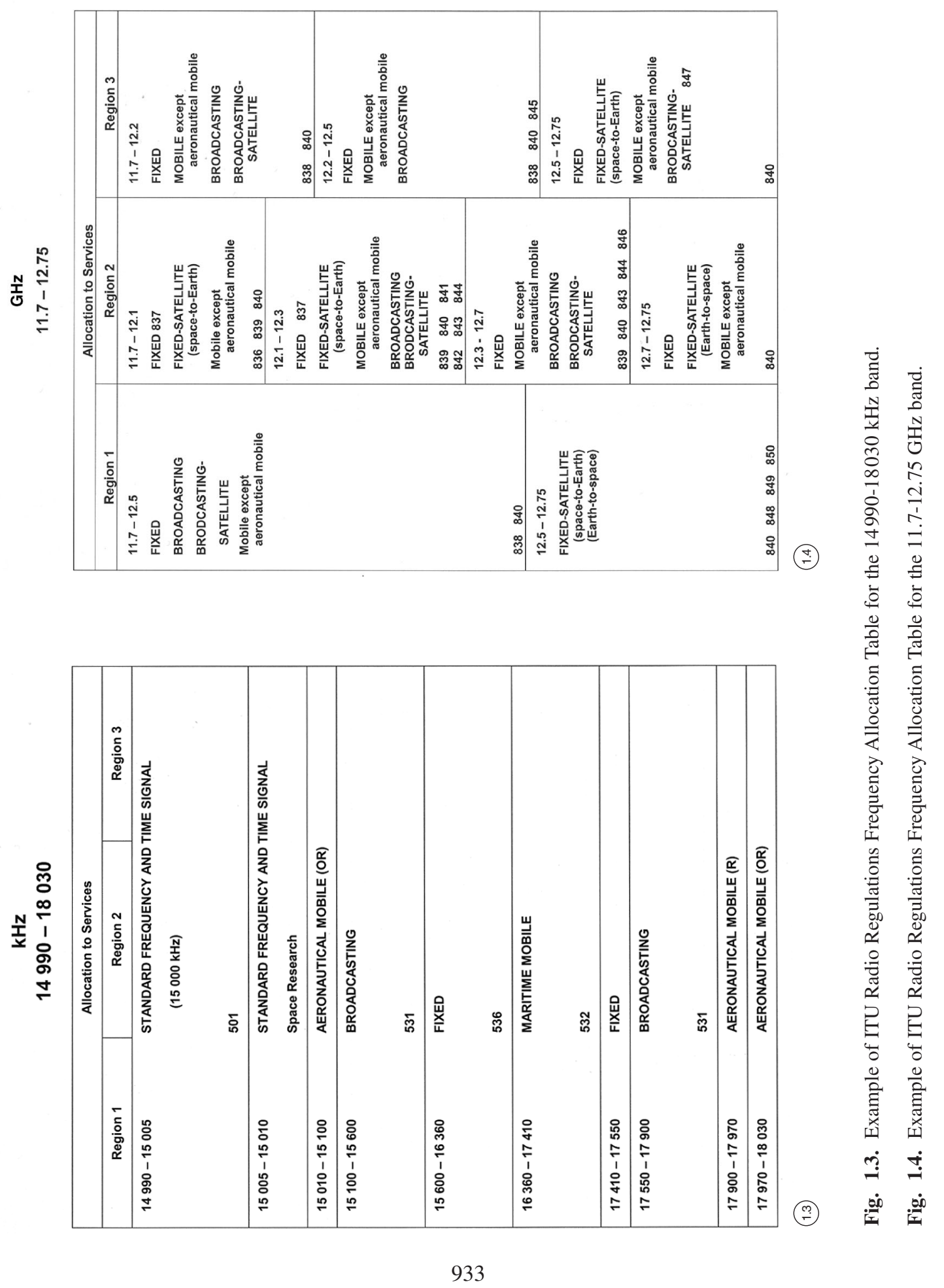
several numbers relate to footnotes not reproduced here detailing specific applied transmission conditions. At higher frequencies, in this case illustrated by the example of fig. 1.4 for the 11.7-12.75 $\mathrm{GHz}$ band allocated mainly to the satellite and fixed satellite broadcasting services where simultaneous worldwide coverage is not achieved, important differences in the bands allocated to the separate services are evident.

Despite the detailed changes that are introduced in such tables from time to time, some general remarks can be made regarding spectral usage for different broad frequency ranges. Radiocommunication at VLF is limited by the available bandwidth, but since ionospheric attenuation is very low, near worldwide coverage can be achieved. Unfortunately the radiation of energy is difficult at such frequencies and complex transmitting antenna systems coupled with large transmitter powers are needed to overcome the high received atmospheric noise background. Because of the stability of propagation, very low frequencies may be used for the transmission of standard time signals and for continuous wave navigation systems which rely on direction-finding techniques, or on phase comparisons between spaced transmissions as in the Omega system operating in the $10-14 \mathrm{kHz}$ frequency range (Pierce et al., 1964), though nowadays more and more such systems are giving way to more reliable satellite services providing the same types of information.

At LF increased propagation losses limit area coverage, but simpler antenna systems are adequate and lower transmitter powers can be employed because of the reduced atmospheric noise. Low-frequency systems are used for communication by on-off keying and frequency-shift keying. Propagation conditions are more stable than at higher frequencies because the ionosphere is less deeply penetrated. Other LF systems relying principally on the ground wave, but which are sometimes degraded by the sky-wave at night, include the Decca continuous wave navigation system $(70-130 \mathrm{kHz})$ now little used, the Loran C pulse navigation system (100 kHz) and long-wave broadcasting (Hall, 1947; Bradley, 1984; Davies, 1990).

At MF daytime absorption is so high as to completely suppress the sky wave. Some use is made of the sky wave at night-time for broadcasting, but generally medium frequencies are employed for ground-wave services (Bradley, 1984).

Despite the decline in use of HF by the fixed service since the early 1970's with the advent of communication satellites and wideband submarine cables, high frequencies continue to be employed predominantly for broadcasting, fixed and mobile point-to-point communications via ionosphere reflection. Table 1.I shows the relative fractions of spectrum allocated to the fixed, sound broadcasting and mobile services.

Maritime elements include coast stations, ship-station radiotelephone working to coast stations and inter-ship working. Aeronautical systems include single-sideband HF radiotelephone links between aircraft and the ground in the aeronautical mobile channels of the $2-22 \mathrm{MHz}$ band and radioteletype links between ground terminals in the aeronautical fixed channels in the $2.5-30 \mathrm{MHz}$ band. The majority of long-distance fixed circuits nowadays rely on satellites and cables for both civilian and military applications. Likewise, there has in recent years been a growth in the deployment of satellites for aeronautical, maritime and land-mobile communications and an explosion in their use for personal mobile phone and location determination applications. Nonetheless, increases in the numbers involved mean that there are actually more HF circuits in use than, say, 20 years ago. Par-

Table 1.I. Approximate fractional usage by the different radio services of the HF-bands.

\begin{tabular}{lr}
\hline \hline Fixed service & $55 \%$ \\
Land and maritime mobile services & $15 \%$ \\
Sound broadcasting service & $15 \%$ \\
Aeronautical mobile service by the amateur service, & $10 \%$ \\
the standard frequency and time service and space research services & $5 \%$ \\
\hline
\end{tabular}


ticularly for military purposes, HF systems are regarded as providing a necessary backup service to fixed links primarily established by other means.

VHF communication by ionospheric scatter propagation between ground-based terminals is possible. Two-way error-correction systems with scattering from intermittent meteor trains can be used at frequencies of 30-40 MHz over ranges of 500-1500 km. Bursts of high-speed data of about $1 \mathrm{~s} \mathrm{du}-$ ration and duty cycles of the order of $5 \%$ can be achieved with transmitter powers of about $1 \mathrm{~kW}$. Forward-scatter communication systems at frequencies of 30-60 MHz, also operating over ranges of about $1000 \mathrm{~km}$, rely on coherent scattering from field-aligned irregularities at a height of about 85 $\mathrm{km}$ in the $D$-region. They are deployed principally at low and high latitudes. Signal intensities are somewhat variable, depending on the incidence of irregularities. Directional transmitting and receiving antennas with intersecting beams are required. Frequency-modulation techniques involving timedivision multiplex are used to combat Doppler effects. Systems with 16 channels, automatic error correction and operating at 100 words per minute, now exist.

Inspection of the Article 8 tables confirms that on Earth-space paths there is an evident dominance of broadcasting, communication and navigation and earth-surveillance satellite channels. As specific examples of such usage the $L$-band navigation service, the land-mobile communication satellite service at $L$ - and $S$-bands, the low frequency remote sensing satellite service at $P$ - and $L$-bands and altimetry at $S$ - and $\mathrm{Ku}$-bands all experience ionospheric propagation effects which are important to characterize for system design and operation.

\subsubsection{Likely future usage}

As the nations of the world become more industrialised, as populations grow and as expectations of standards of living rise, demand for radiocommunication services will increase. Travel delays too mean that future trends will see more personal point-to-point communication contacts as journey times rise and traffic grid-locks become more common. All the world is seeking access to a mobile phone, a television set and an Internet link. At the same time there must be increases in levels of surveillance and civil protection. So the radio spectrum is an asset of growing importance to industry, personal communications and civil protection. Yet it is of finite size, and despite the moves towards ever-increasing frequencies, the next decade will see significant increases in radio usage. Therefore to avoid or minimise congestion, spectrum management techniques must keep abreast of demand.

Current procedures for frequency allocation and the associated national assignment arrangements within the terms of the ITU Radio Regulations are known to be grossly inefficient. Demand and available spectral bands adopted at Radio Conferences, held from time to time to review and update these regulations, are generally poorly matched despite the earnest endeavours of those involved to produce equitable solutions. There is little grasp of the capacity of the spectrum. Attempts at devising procedures to ensure service reliability and compatibility protection from co-channel and adjacent channel services are usually subservient to meeting all stated requirements. The inertia of the system mitigates against optimising spectrum utilisation and there is lack of flexibility to respond to changing requirements. Many assignments are under-employed with operators demanding unrestricted full-time use of these, whereas in practice they are required for only a fraction of this. Existing ITU frequency allocations are too restrictive and inhibit technological changes. At the same time many transmitters perform with poor characteristics: grossly too high powers, inefficient antennas with too wide beams or sidelobes and unnecessary emission characteristics such as bandwidths and spurious emissions. Multiple radiation of the same information, on separate frequencies, duplication in time or from separate sites is common.

To respond to this situation, moves are afoot in many countries to develop alternative approaches to frequency management (Bradley, 2002). These aim to bring a sense of awareness to 
radio operators to optimise their requirements and emission characteristics by means of financial incentives. For example the switch from an analogue to a digital television service, besides the improvement in reception quality, means that 6 digital channels can be accommodated within the same bandwidth. Charges are being introduced for licences, in principle set at levels required to achieve the desired effect, rather than to optimise exchequer input. There are three ways in which licenses can be awarded: sold at pre-determined costs; auctioned, or acquired by secondary trading. As examples of the auctioning approach, in the year 2000 several European governments auctioned frequencies for the introduction of $3 \mathrm{G}$ mobile licences. Secondary trading can enhance efficiency and flexibility, aiding time sharing within a regulatory framework. Future licenses should be of finite duration of just a few years, rather than open-ended as has usually been the case with past procedures.

At this stage it is impossible to anticipate what new radio services the next decades will bring, except to suppose in general terms that there will be more demand for communications within the home: greater automatic control of functions with remote monitoring and over-ride, and provision of additional data services. The use of personal positioning devices seems likely to become much more widespread, so that the locations of individuals are always known. European companies have invested huge fortunes in licenses for the so-called «next generation» $3 \mathrm{G}$ mobile communication systems, in the anticipation that new requirements will need far greater bandwidths than has hitherto been possible. But if agreement is reached on how to re-organise spectral utilisation on a more dynamic, flexible and cost-effective way, the door will be open for such innovative procedures to be developed matched to user demands.

A naive viewpoint is that future adaptive radio systems will probe the propagation medium in real-time and then optimise their transmission characteristics; hence there is no need for further propagation data. But just as spectrum usage has to be internationally controlled, so the latest adaptive systems must be engineered to operate within defined bounds that themselves have to be specified from a knowledge of the radio medium. More advanced systems require propagation information that has hitherto not been necessary. Whilst understandably greatest interest centres on UHF and higher frequencies with the greater available bandwidths, where tropospheric impairments dominate, there remain large numbers of terrestrial radio services still relying on the ionosphere for support. As examples, at HF it was hitherto usual to seek to model received signal-noise ratios and their statistical variations, but the importance to digital systems of being able to characterise likely time spreads and Doppler dispersions has been clearly demonstrated. Ways of short-term forecasting of ionospheric changes, particularly those concerned with ionospheric storms remain needed.

Earth-space links up to SHF too can be significantly affected by the ionospheric presence (ITU-R P531-7, 2003). A priority must be the improved modelling of propagation effects on Earth-space links. Many of these effects are related to the columnar Total Electron Content (TEC) between spacecraft and ground receiver, including refraction, dispersion, angular deviation, change of plane of polarisation and group delay. Existing international models of median TEC and its day-to-day variability are grossly inadequate in meeting requirements of latest generation communication and surveillance systems. At the same time ionospheric scintillation imposes a major problem to satellite systems, particularly for equatorial and high-latitude paths. Scintillation models need to be much improved by extension to provide a broader temporal and spatial coverage than hitherto.

\subsection{Propagation PHENOMENA WHICH UNDER SOME CIRCUMSTANCES CAN LEAD TO RADIO-SYSTEM IMPAIRMENTS AND THAT HAVE BEEN MODELLED WITHIN THE COST 271 WORK PROGRAMME}

The later sections of this Report discuss the various models that have been generated within the Action. Here we summarise the individual propagation impairments that can arise under 
Table 1.II. Estimated maximum ionospheric effects at $1 \mathrm{GHz}$ for elevation angles of about $30^{\circ}$ one-way traversal (ITU-R P531-7, 2003).

\begin{tabular}{llc}
\hline \multicolumn{1}{c}{ Effect } & Magnitude & Frequency dependence \\
\hline Faraday rotation & $108^{\circ}$ & $1 / f^{2}$ \\
Propagation delay & $0.25 \mu \mathrm{s}$ & $1 / f^{2}$ \\
Refraction & $<0.17 \mathrm{mrad}$ & $1 / f^{2}$ \\
Variation in direction of arrival & $0.2 \mathrm{~min}$ of arc & $1 / f^{2}$ \\
Absorption (polar cap) & $0.04 \mathrm{~dB}$ & $\sim 1 / f^{2}$ \\
Absorption (auroral + polar cap) & 0.05 & $\sim 1 / f^{2}$ \\
Absorption (mid-latitude) & $<0.01 \mathrm{~dB}$ & $1 / f^{2}$ \\
Dispersion & $0-4 \mathrm{~ns} / \mathrm{MHz}$ & $1 / f^{3}$ \\
Scintillation & (See ITU-R P531-7, 2003, Sec. 4) & \\
\hline
\end{tabular}

some circumstances. A radio wave is specified in terms of five parameters: its amplitude, phase, direction of propagation, polarisation and frequency. The principal effects of the ionosphere in modifying these parameters are considered as follows (Bradley, 1996; Cannon and Bradley, 2003).

\subsubsection{Refraction}

The change in direction of propagation resulting from the traverse of a thin slab of constant ionisation is given approximately by Bouger's Law in terms of the refractive index and the angle of incidence. A more exact specification including the effects of the Earth's magnetic field is provided by the Haselgrove equation solution (Haselgrove, 1954). The refractive index is determined from the Appleton-Hartree equations of the magnetoionic theory as a function of the electron density and electron-collision frequency, together with the strength and direction of the Earth's magnetic field, the wave direction and the wave frequency (Ratcliffe, 1959; Budden, 1961). The dependence on frequency leads to wave dispersion of modulated signals. Since the ionosphere is a doubly-refracting medium it can transmit two waves with different polarisations (see below). The refractive indices appropriate to the two waves differ. Refraction is reduced at the greater wave frequencies, and at VHF and higher frequencies it is given approximately as a function of the ratio of the wave and plasma frequencies, where the plasma frequency is defined in terms of a universal constant and the square root of the electron density. Table 1.II lists estimates of the magnitude of the refraction and of other propagation parameters for signals at a frequencv of $1 \mathrm{GHz}$ which traverse the whole ionosphere one way with an elevation angle of $30^{\circ}$.

\subsubsection{Change in phase-path length}

The phase-path length is given approximately as the integral of the refractive index with respect to the ray-path length. Ignoring spatial gradients, the change in phase-path length introduced by passage through the ionosphere to the ground of signals at VHF and higher frequencies from a spacecraft is proportional to the columnar total-electron content. 


\subsubsection{Group delay}

The group and phase velocities of a wave differ because the ionosphere is a dispersive medium. The ionosphere reduces the group velocity and introduces a group delay which for transionospheric signals at VHF and higher frequencies, like the phase-path change, is proportional to the total electron content.

\subsubsection{Polarisation}

Radio waves that propagate in the ionosphere are called characteristic waves. There are always two characteristic waves known as the ordinary wave and the extraordinary wave; under certain restricted conditions a third wave known as the $Z$-wave can also exist. In general the ordinary and extraordinary waves are elliptically polarised. The polarisation ellipses have the same axial ratio, orientations in space that are related such that under many conditions they are approximately orthogonal, and electric vectors which rotate in opposite directions. The polarisation ellipses are less elongated the greater the wave frequency. Any wave launched into the ionosphere is split into characteristic ordinary and extraordinary wave components of appropriate power. At MF and above these components may be regarded as travelling independently through the ionosphere with polarisations which remain related, but continuously change to match the changing ionospheric conditions. The phase paths of the ordinary and extraordinary wave components differ, so that in the case of transionospheric signals when the components have comparable amplitudes, the plane of polarisation of their resultant slowly rotates. This effect is known as Faraday rotation.

\subsubsection{Absorption}

Absorption arises from inelastic collisions between the free electrons, oscillating under the influence of the incident radio wave, and the neutral and ionised constituents of the atmosphere. The absorption experienced in a thin slab of ionosphere is given by the Appleton-Hartree equations and under many conditions is proportional to the product of electron density and collision frequency, inversely proportional to the refractive index and inversely proportional to the square of the wave frequency. The absorption is referred to as non-deviative or deviative depending on whether it occurs where the refractive index is close to unity. Normal absorption is principally a daytime phenomenon. At frequencies below $5 \mathrm{MHz}$ it is sometimes so great as to completely suppress effective propagation. The absorptions of the ordinary and extraordinary waves differ, and in the range $1.5-10 \mathrm{MHz}$ the extraordinary wave absorption is significantly greater.

\subsubsection{Amplitude fading}

If the ionosphere were unchanging the signal amplitude over a fixed path would be constant. In practice, however, fading arises as a consequence of variations in propagation path, brought about by movements or fluctuations in ionization (ITU-R, 1990). The principal causes of fading are: a) variations in absorption; b) movements of irregularities producing focusing and defocusing; c) changes in path length among component signals propagated via multiple paths; d) changes in polarisation, such as for example due to Faraday rotation.

These various causes lead to different depths of fading and a range of fading rates. The slowest fades are usually those due to absorption changes which have a period of about $10 \mathrm{~min}$. The deepest and most rapid fading occurs from the beating between two signal components of comparable am- 
plitude propagated along different paths. A regularly reflected signal together with a signal scattered from spread- $F$ irregularities can give rise to so-called flutter fading, with fading rates of about $10 \mathrm{~Hz}$. A good general survey of fading effects, including a discussion of fading statistics, has been produced. On operational communication circuits fading may be combated by space diversity or polarisation-diversity receiving systems and by the simultaneous use of multiple-frequency transmissions (so-called frequency diversity).

\subsubsection{Frequency deviations}

Amplitude fading is accompanied by associated fluctuations in group path and phase path, giving rise to time and frequency-dispersed signals. When either the transmitter or receiver is moving, or there are systematic ionospheric movements, the received signal is also Doppler-frequency shifted. Signals propagated simultaneously via different ionospheric paths are usually received with differing frequency shifts. Frequency shifts for reflections from the regular layers are usually less than $1 \mathrm{~Hz}$, but shifts of up to $20-30 \mathrm{~Hz}$ have been reported for scatter-mode signals at low latitudes.

\subsubsection{Reflection, scattering and ducting}

The combined effect of refraction through a number of successive slabs of ionisation can lead to ray reflection. This may take place over a narrow height range as at LF, or rays may be refracted over an appreciable distance in the ionosphere as at HF. Weak incoherent scattering of energy occurs from random thermal fluctuations in electron density, and more efficient aspect-sensitive scattering from ionospheric irregularities gives rise to direct backscattered and forward-scatter signals. Ducting of signals to great distances can take place at heights of reduced ionisation between the $E$ - and $F$-regions, leading in some cases to round-the-world echoes (Fenwick and Villard, 1963). Ducting can also occur within regions of field-aligned irregularities above the maximum of the $F$-region (Nielson, 1968).

\subsubsection{Scintillation}

Scintillations are variations of amplitude, phase, polarisation and angle-of-arrival produced when a radio wave passes through electron density irregularities in the ionosphere (Ratcliffe,1956). Ionosphere scintillations present themselves as fast fluctuations of signal level with peak-to-peak amplitude fluctuations from $1 \mathrm{~dB}$ to over $10 \mathrm{~dB}$ and lasting for several minutes to several hours. The phenomena are caused by one of two types of ionosphere irregularities: sufficiently high electron density fluctuations at scale sizes comparable to the Fresnel zone dimension of the propagation path, or sharp gradients of ambient electron density, especially in the direction transverse to the direction of propagation (ITU-R P531-7, 2003).

Either type of irregularity is known to occur in the ionosphere under certain solar, geomagnetic and upper atmospheric conditions, and the scintillations can become so severe that they represent a practical limitation for communication systems. Scintillations have been observed at frequencies from about $10 \mathrm{MHz}$ to about $12 \mathrm{GHz}$. For systems applications, scintillations can be characterised by the fading depth and period. A useful index to quantify the severity of scintillation is the scintillation index $S_{4}$, which is defined as the standard deviation of received power divided by the mean value of the received power. The fading period of scintillation varies over quite a large range from less than one tenth of $1 \mathrm{~s}$ to several minutes, as the fading period depends both upon the apparent motion of the irregularities relative to the ray path, and in the case of strong scintillations, on its severity. The fading period of gigahertz scintillation ranges from approximately 1 to $10 \mathrm{~s}$. Long period (of the or- 
der of tens of seconds) components of saturated scintillation $\left(S_{4}\right.$ approaching 1$)$ at VHF- and UHFbands have also been observed.

Ionosphere scintillations exhibit a wide range of variations in frequency dependence, morphology patterns, and diurnal, seasonal and solar-cycle dependence. Typically $S_{4}$ varies as frequency raised to the inverse power of 1.5 , though on different occasions inverse power frequency dependences in the range 1 to 6 have been encountered. During an ionosphere scintillation event, the Nakagami density function provides a close match to the statistics of the instantaneous variation of amplitude. For zenith angles of the propagation path up to $70^{\circ}$ the $S_{4}^{2}$ is proportional to the secant of the zenith angle. Scintillation depth also depends on local time, season and longitude as defined by the angle between the sunset terminator and the local magnetic meridian at the apex of the field line passing through the line-of-sight at the height of the irregularity slab. There are also important latitudinal differences in the incidence of scintillations, with these being particularly strong at equatorial latitudes where they are caused by plasma bubbles, and at high latitudes, related to auroral and geomagnetic activities. At mid-latitudes, ionosphere scintillation tends to be associated with the incidence of the phenomenon known as spread- $F$.

\subsubsection{Examples of operational problems within the European theatre (Bishop, 2003)}

From the foregoing it is evident that a series of different propagation phenomena are important to the different radio services, ranging from the requirement to be able to predict, both in the long-term and in near-real-time, the conditions under which ionospheric support is possible and the intensities of the resulting signals for communications at $\mathrm{HF}$ and below, to more stringent requirements on Earth-space links where the needs of navigation and surveillance dictate that changes from free-space values of group path, angle-of-arrival, polarisation and scintillation all be quantifiable.

The dominant ionospheric impairments to $L$-band navigation systems are considered to arise from delay effects, with scintillations giving secondary problems. Land mobile communication satellite systems are principally affected by scintillations and also to some extent by Faraday rotation effects. Low-frequency earth observation sensors are mainly influenced by Faraday rotation effects, but also by delay uncertainties. Delay uncertainties are understandably of greatest importance to altimetry systems such as the European RA-2 system for determining ocean surface heights using a pulsed radar, with also resolution limited by dispersion effects. Mobile satellite communications using the $L$ - and $S$-bands are subject to the variable effects of phase and amplitude scintillations as well as to a lesser extent to Faraday rotation problems, though in practice most systems avoid the latter difficulties by using circularly polarised signals. Their main need is for accurate global scintillation models.

The European Space Agency (Arbesser-Rastburg, 2001) is implementing a satellite navigation system EGNOS (European Geostationary Navigation Overlay System). This involves a network of ground-based stations measuring slant pseudo-range errors from satellites in a $20000 \mathrm{~km}$ orbit. TEC values and error budgets determined from multi-band reception are broadcast so that mobile users can correct positioning errors, even when employing single-frequency reception. At the present time though there are needs to know the maximum ionisation gradients in space and time that can arise and the degree of «obliquity errors». This points to the need of having reliable 4D electron-content models, including a fast integration tool to determine slant TEC. "Worst-case» statistics are needed for input to an end-to-end simulator, to be derived from ionospheric conditions under storm and TID scenarios. What are the dynamics of the ionosphere and can scintillations cause receivers to loose lock? There are longer-term needs to be able to predict trans-ionospheric propagation through planetary ionospheres, such as that of Mars. The requirements of the various listed services for ionosphere characterization, not otherwise met, are seen to be many. The work that has been carried out within COST 271 was responsive to many of these various needs to the 
maximum extent that it was possible to do so within the available project resources and designated time-scales.

\subsection{SPACE WEATHER PARAMETERS THAT CREATE IMPACT ADVERSELY AND SIGNIFICANTLY ON RADIO PROPAGATION CHARACTERISTICS}

In this section brief consideration only is possible to identify the different phenomena that arise under separate conditions with different time scales to affect the ionosphere, that are pertinent to the specific discussions of the remaining seventeen other Work packages.

\subsubsection{Solar flares}

These are sudden, relatively short-lived brightenings of localized «plage» regions of the solar chromosphere. They have life-times ranging from a few minutes up to several hours, with an average duration of around half an hour. The enhanced electromagnetic and associated particle radiation emitted during a flare can affect the terrestrial and interplanetary environment. Not all flares yield particle streams, consisting mainly of fast moving protons and electrons, which are detectable on the Earth; this depends on the position on the Sun where the eruption occurs relative to the Earth's direction. Flares are classified according to their importance, or area at maximum brightness, and by a qualitative assessment of brightness. Associated simultaneously with a flare are radio noise bursts and sudden ionospheric disturbances. Delayed effects detectable at the ground anything from 15 min to $72 \mathrm{~h}$ afterwards are the onset of polar cap absorption, magnetic storms, ionospheric storms and aurorae.

\subsection{2. $X$-ray enhancements}

Increased radiation at radio and $X$-ray frequencies associated with a flare is often of sufficient intensity to affect the Earth's environment. In general effects are most pronounced with major solar flares, but sometimes likewise pronounced effects can occur with sub-flares. Increases in solar $X$-ray and also ultra-violet radiation associated with a flare lead to sudden ionospheric disturbances on time scales ranging from minutes to several hours, during which there is enhanced absorption and frequency deviation of HF signals, while at the same time there is amplitude enhancement and phase change of VLF and LF signals. A number of other features are also associated with sudden ionospheric disturbances, including short-wave fadeouts and sudden enhanced cosmic noise absorption events. Ionospheric storms are closely associated with geomagnetic storms and lead to reduced $F 2$-region maximum ionization densities and associated $\mathrm{HF}$ circuit maximum usable frequencies.

\subsubsection{Coronal mass ejections and magnetic effects}

Extremely high energy solar particles emitted during a disturbance travel at velocities approaching the speed of light and may arrive at the Earth after delays of from $15 \mathrm{~min}$ to an hour. There they cause polar cap absorption events which, though relatively infrequent, can persist typically up to $20 \mathrm{~h}$. Polar cap absorption arises from the deflection by the Earth's magnetic field of the incident particle streams to the polar regions of the Earth where they cause enhanced ionization in the ionospheric $D$-region. This gives rise to severe absorption of HF waves propagated across the polar regions which can persist for several days. So HF circuit lowest usable frequencies are increased, with the consequence that 
the usable frequency band is reduced, or even propagation is not possible at all. These events are normally followed by disturbances in the geomagnetic field. More common low to medium energy protons and electrons cause magnetic and ionospheric storms and the onset of the aurorae.

Geomagnetic storms arise from low energy plasma emitted from the Sun. Normally a geomagnetic storm comprises three phases: a) an initial phase in which a sudden initial disturbance (sudden commencement) quickly subsides and is followed by a quiet period lasting several minutes or hours; b) a main phase with an increase in the disturbance which persists for several hours or days, and c) a recovery phase with a gradual return of the field intensity to normal. Various indices are used to describe the magnitude of the disturbance, the most common of which are the $K$ index and the $A p$ value.

Auroral activity is a visible indication of disturbances in the Earth's upper atmosphere. It occurs most frequently in the auroral zones in the northern and southern hemispheres. These are roughly circular bands around the geomagnetic poles at the centre of which there is a maximum of intensity. Auroral induced ionization is associated with increased radio-wave absorption and scattering of $\mathrm{HF}$ waves. It occurs quite commonly, particularly during periods of enhanced solar activity during the upper part of the solar cycle.

\subsubsection{Solar radio burst emissions}

Radio emission from the Sun has three distinct components, originating from the quiet sun, from bright regions and from transient disturbances, such as flares. Most flares are accompanied by increased emission at radio frequencies. The intensity and complexity of the radiation generally increase with the flare importance. The eruption of particle streams through the Sun's atmosphere is believed to be the cause of this increased emission. Radio burst data are an important pre-cursor and indicator of possible solar particle emissions.

\subsection{WORK DIRECTED TOWARDS IMPROVED PROPAGATION MODELS}

Various propagation models are available for different frequency bands and geographical theatres as described in current ITU Recommendations. For the most part they are based on a monthly median ionosphere and provide estimates of monthly median propagation parameters, together with some measure of the statistical probabilities of variations about these conditions. COST 271 has worked to contribute to state of the art with a number of specialist Workshops and an impressive list of publications. Following from earlier studies carried out within Actions COST 238 (Bradley, 1999) and COST 251 (Hanbaba, 1999) aimed at developing and testing different long- and short-term ionosphere and propagation models for varying applications relevant to radio usage and ITU long-term service planning, topics examined relating to the impact of space weather on communications have embraced, among other aspects, «nowcasting» and forecasting techniques. Specifically, web-based services for disseminating near-real-time space weather, ionospheric model and radio propagation predictions have been established by a number of participating organizations. These are described in greater detail elsewhere in this Report.

An important aspect of the work has been the review of existing propagation models, as well as the development of new models. The different studies that have been undertaken may be summarized as investigations of linear and non-linear ionospheric predictions; radio occultation techniques (using LEO satellites to determine height profiles of electron density in remote areas); tomographical techniques (using signals from orbiting spacecraft to give 3D reconstructions of electron density, also including in remote areas; ionisation changes at different heights, including above the height of peak density, using various probing methods; horizontal gradients and structures of TEC; geomagnetic storms and their impact on the ionosphere; ionospheric irregularities with various scale sizes; ionos- 
pheric variability; extreme case scenarios; amplitude and phase scintillations, ionospheric coherence on Earth-space links; propagation errors and noise on terrestrial and space-space communication systems and on Earth-space navigation systems.

Future radio system design, spectrum utilisation strategies and spectrum management procedures require propagation information not currently available. Collaborative European research project COST 271 has come some ways to meet these needs.

\subsection{Mitigation techniQueS}

Within the context of COST 271 «mitigation techniques» are interpreted as means of reducing propagation impairments to radio systems. The basic general approaches possible are well known (Bishop et al., 1996). These include routing via other propagation paths to avoid spatially limited sources of impairment, transmitting at a different time, use of a totally different radio system probably with a markedly separate operational frequency, frequency agility to avoid narrow-band interference - either changes of carrier frequency or in-band frequency variability, use of digital as opposed to analogue systems to minimise overall bandwidths, frequency, time, polarisation and/or spatial diversity systems and use of adaptive systems in any of the above regards, as well as with varying data transmission rates.

A radio user with a particular system seeks guidance on how to optimise his system (Bishop, 2003), and in many cases the optimum solution will be specific to him, depending on precisely what are the operational scenario, constraints, importance and resources available so that a tailor-made solution, perhaps involving a combination of the above approaches is what is needed. From a user's standpoint there are three separate aspects: i) how to engineer a particular system to meet a defined requirement, ii) how to best operate an established system on any given occasion, and iii) to receive advance warning of abnormal propagation conditions and advice on what to do regarding these.

Space-weather parameters can be classified as direct if they are regularly monitored and are an established indicator of ionospheric state and indirect if they are part of the chain of events, but do not in themselves characterise the state of the ionosphere. Other COST 271 WP's have made use of indirect parameters in their studies and used direct parameters to generate corresponding same epoch and forecast ionospheric maps and models and the associated propagation models. Evidently user synergy is needed to ensure that these propagation models provide the types of information that are wanted for the implementation of the most appropriate mitigation techniques.

\subsection{FEEDBACK FROM THE USER COMMUNITY}

Inevitably, work of the nature of that undertaken within COST 271, by its great diversity and range of topics considered, will be incomplete. Interaction with as representative and extensive as possible a group of informed radio users is seen as essential to assessing the practical improvements that can be achieved with the use of its products. To identify areas of continued incomplete knowledge is also important to scientists and engineers working within allied research programmes. In other words, this section is an overview to help the reader appreciate and understand the scope of the work undertaken.

\section{REFERENCES}

ARBESSER-RASTBURG, B. (2001): User requirements for trans-ionospheric propagation modelling, draft paper presented at COST271 First Management Committee Meeting, Abdus Salam ICTP, Trieste (Italy), 24-28 January 2001. 
Bishop, G.J., A.J. Mazzella, E.A. Holland and G.S. Rao (1996): An overview of ionospheric effects and mitigation in RF communication, navigation and surveillance, in Ionospheric Effects Symposium, IES-96, 7-9 May 1996, Alexandria (VA).

BisHOP, G.J. (2003): Space environmental impacts on radio system operations, in COST 271 Action, 23-27 September 2003, Spetses, Greece (published on line at the COST 271 Web site).

BRADLEY, P.A. (1984): The ionosphere and radio communications, in Links for the Future - Science, Systems and Services for Communications, edited by P. DEWILDE and C.A. MAY (IEEE/Elsevier Science), 171-174

Bradley, P.A. (1996): Ionospheric effects on radio signals, in Principles of Radio Communication, edited by F. MAZDA (Butterworth-Heinemann, Oxford), 8-12.

BRADLEY, P.A. (1999): Prediction and retrospective ionospheric modelling over Europe, in COST Action 238 Prime Final Report, Commission of the European Communities, U.K.

Bradley, P.A. (2002): Spectrum utilization, spectrum management and COST 271, Acta Geod. Geophys. Hung., 37 (4), 419-423.

BrAdLEy, P.A. (2003): 3rd COST 271 Workshop Proceeding on Significant results in COST 271 Action - A review, contribution to discussions at 5th COST 271 Management Committee Meeting, 27 February-1 March, Roma.

Budden, K. (1961): Radio Waves in the Ionosphere (Cambridge University Press).

CAnnon, P. and P.A. Bradley (2003): Ionospheric propagation, in Propagation of Radiowaves, edited by L.W. BARCLAY (Institution of Electrical Engineers, London), 2nd edition, 335-355.

DAVIES, K. (1990): Ionospheric radio, in Electromagnetic Wave Series 31 (Peter Peregrinus Ltd., Institution of Electrical Engineers, London).

FENwick, F.B. and O.G. VILLARD (1963): A test of the importance of ionosphere-ionosphere reflections in long distance and around-the-world high frequency propagation, J. Geophys. Res., 68, 5659-5666.

HALL, J.S. (1947): Radar aids to navigation, in Radiation Laboratory Series 2 (McGraw-Hill, New York).

HANBABA, R. (1999): Improved quality of service in ionospheric telecommunication systems planning and operation, in COST 251 Prime Final Report, Commission of the European Communities (Space Research Center, Warsaw, Poland).

HASElgrove, J. (1954): Ray theory and a new method for ray tracing, in Reports on Conference on the Physics of the Ionosphere (Physical Soc., London), p. 355.

ITU (2004): Radio Regulations (International Telecommunication Union, Geneva).

ITU-R (1990): Ionospheric propagation and noise characteristics pertinent to terrestrial radiocommunication systems design and service planning (Fading), Report 266-7 (International Telecommunication Union, Geneva).

ITU-R P531-7 (2003): Ionospheric Propagation Data and Prediction Methods Required for the Design of Satellite Services and Systems (International Telecommunication Union, Geneva).

NIELSON, D.L. (1968): The importance of horizontal $F$-region drifts to transequatorial VHF propagation, in Scatter Propagation of Radio Waves, edited by E.V. Thrane, AGARD Conference Proceedings 37, NATO, Neuilly-sur-Seine, France.

Pierce, J.A., W. PAlmer, A.D. Watt and R.H. WoOdward (1964): Omega a worldwide navigational system (Pickard and Burns Electronics), $P \& B$ Publication No. 886.

RATCLIFFe, J.A. (1956): Some aspects of diffraction theory and their application to the ionosphere, in Reports on Progress in Physics (Physical Soc., London), 19, p. 266.

RATCLIFFE, J.A. (1959): The magnetoionic Theory (Cambridge University Press).

TulunAY, Y.K. (2001): WP 1.1. Objectives-Minutes of the First Management Committee of COST 271, 24-28 January 2001, Abdus Salam ICTP, Trieste (Italy). 